\title{
W/Z Production at ATLAS
}

\section{Sara Borroni, on behalf of the ATLAS Collaboration*}

Sapienza Universitá and INFN Roma

E-mail: sara.borroniecern.ch

The $\mathrm{W}$ and $\mathrm{Z}$ bosons are produced at LHC with high cross-sections and are the most copious sources of high- $\mathrm{p}_{T}$ leptons. These two characteristics make them suitable for many purposes, from the very first stages of the data taking up to the high luminosity scenarios. In particular $\mathrm{Z}$ to lepton pairs events allow to determine lepton trigger and reconstruction efficiencies as well as resolutions and relative alignment. Then $\mathrm{W}$ and $\mathrm{Z}$ cross-sections measurement is one of the first benchmarks to have a complete and detailed understanding of the known Standard Model physics in new experimental conditions, since high- $\mathrm{p}_{T}$ decays in leptons have the clearest experimental signature. They also allows to estimate with good precision the data luminosity. Finally measurement of the decay lepton spectra from $\mathrm{W}$ production gives information on the gluon PDF at low- $\mathrm{x}$ and can thus improve knowledge of PDFs.

XVIII International Workshop on Deep-Inelastic Scattering and Related Subjects, April 19 - 232010

Florence, Italy

\footnotetext{
* Speaker.
} 


\section{Introduction}

Due to their large cross-sections the $\mathrm{W}$ and $\mathrm{Z}$ bosons are copiously produced at $\mathrm{LHC}$ and are the highest source of high- $\mathrm{p}_{T}$ leptons. In fact at $7 \mathrm{TeV}$ center of mass energy $\sigma \times B R(Z \rightarrow l l) \sim 1$ $\mathrm{nb}$ and $\sigma \times B R(W \rightarrow l v) \sim 9 \mathrm{nb}$ with $l=e, \mu$, which means after selection order of $\sim 2 \times 10^{4}$ $Z \rightarrow l l$ events and $2 \times 10^{5} \mathrm{~W} \rightarrow l v$ events are expected in $100 \mathrm{pb}^{-1}$ of integrated luminosity.

These two characteristics make them suitable for many purposes. In the following will be shown how to use the $\mathrm{Z} \rightarrow l l$ process, which is almost background free, to measure lepton trigger and reconstruction efficiencies as well as resolutions and relative alignment. Then $\mathrm{W}$ and $\mathrm{Z}$ crosssection measurement is illustrated, which can be performed with $\sim 5 \%$ experimental uncertainty with $100 \mathrm{pb}^{-1}$ of integrated data luminosity. Finally how to use the lepton decay spectra from $\mathrm{W}$ production to improve theoretical gluon PDF knowledge in the low- $x$ regime is shown.

\section{Studies of detector performance using $W / Z$ decays}

Detector understanding has already started with the study of the low luminosity $900 \mathrm{GeV}$ center of mass energy data collected in December 2009. The first data and Monte Carlo comparisons show that basic objects performances such as leptons, missing- $\mathrm{E}_{T}$ and tracking (fig.1) are pretty well understood.

At higher luminosity it will be possible to use leptonic $\mathrm{Z}$ decays for more detailed performance assessment. In particular $14 \mathrm{TeV}$ Monte Carlo studies [1] show that a Tag\&Probe method allows to determine lepton trigger and reconstruction efficiencies with a statistical uncertainty of $\sim 5 \%$ and systematic uncertainties at the percent level with $50 \mathrm{pb}^{-1}$ of integrated luminosity. With the same luminosity from the fit to the $\mathrm{Z}$ line-shape is possible to determine the momentum scale of the leptons (from peak position) and the resolution (from the width) at percent level. Moreover the $\mathrm{Z} \rightarrow \mu \mu$ process allows to determine relative alignment between Inner Detector and Muon Spectrometer, which affects muon momentum resolution and the $\mathrm{Z} \rightarrow \tau \tau$ peak position allows the extraction of the missing $\mathrm{E}_{T}$ scale.

\section{Measurement of $\sigma(p p \rightarrow W / Z+X) @ \mathbf{L H C}$}

At LHC, at the leading order, $\mathrm{W} / \mathrm{Z}$ production occurs via $q \bar{q} \rightarrow W / Z$ process. At central rapidity the momentum fraction of the colliding parton lies in the range $10^{-3}<x<0.1$. Given that the gluon is the dominant parton in this range, the production is driven by sea-quark interaction generated by the gluon splitting process. Thus W/Z cross-sections are dominated by the theoretical uncertainties on the gluon distribution at low- $x$.

A robust selection has been developed which allows to measure the W/Z cross-section with an experimental uncertainty $\sim 5 \%$ (plus luminosity $\sim 10 \%$ ) with the first $50 \mathrm{pb}^{-1}$ of data at 14 $\mathrm{TeV}$, which means with $100 \mathrm{pb}^{-1}$ of data at $7 \mathrm{TeV}$ (fig.2). This requires high $\mathrm{p}_{T}$ isolated leptons in the geometrical acceptance, a $20 \mathrm{GeV}$ single lepton trigger, an invariant de-lepton mass between $80<\mathrm{M}_{Z}<100 \mathrm{GeV}$ for $\mathrm{Z}$ search and $\mathrm{E}_{\text {miss }}^{T}>25 \mathrm{GeV}$ and $\mathrm{M}_{T}>40 \mathrm{GeV}$ for $\mathrm{W}$ search. The main contribution to the systematic error is due to QCD background estimation (extracted from data), while the main theoretical uncertainty is just due to the gluon PDF. The uncertainties improved a 

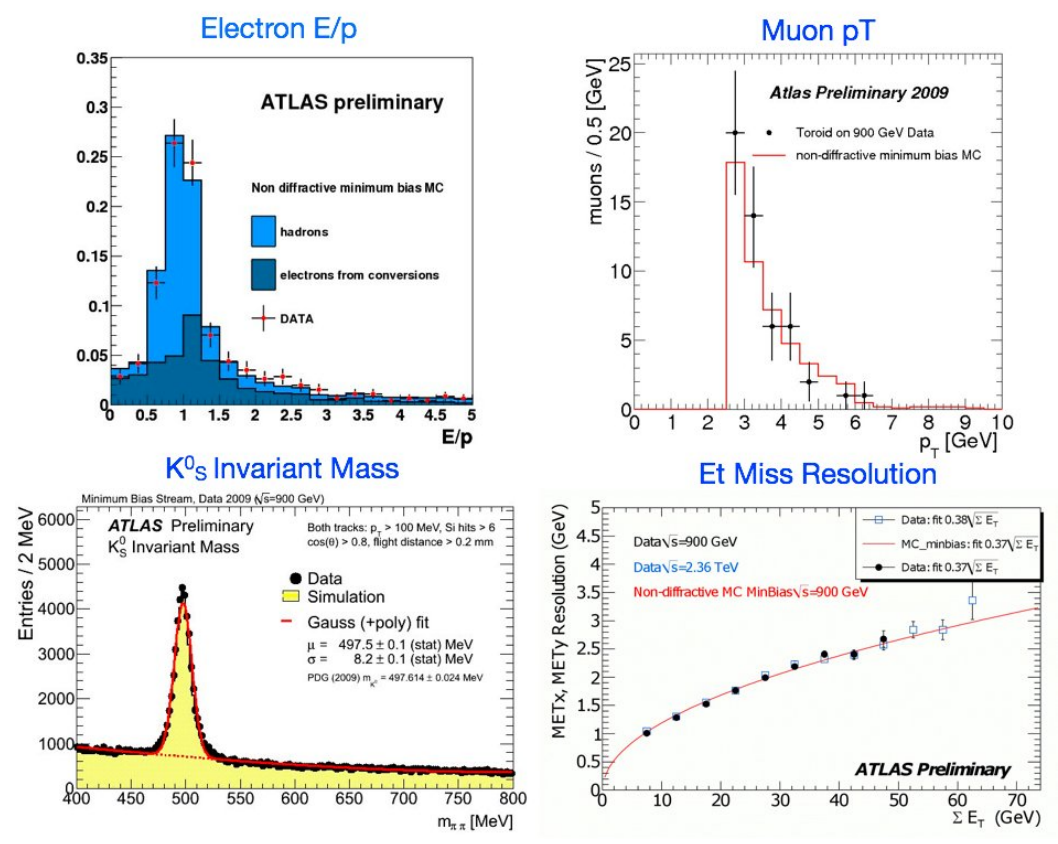

Figure 1: Example of detector performance with $900 \mathrm{GeV}$ center of mass energy data from December 2009 data taking. The plots show data/MC comparisons for basic objects distributions: electrons E/p, muon momentum, di-pion invariant mass (the K-short peak is visible) and transverse energy resolution vs the transverse energy sum in the event.

lot with the input of HERA data, from $16 \%$ to $3.5 \%$ - and more recently $1.5 \%$ [3]. However the most recent data have not yet been fully exploited and PDF uncertainties in the spectra at central rapidity are still large since the spread obtained from using different PDF sets is larger than the uncertainty from each set (see for example http://projects.hepforge.org/mstwpdf/pdf4lhc/). This also implies that it's possible to measure the data luminosity using these processes with no more than $\sim 8 \%$ precision.
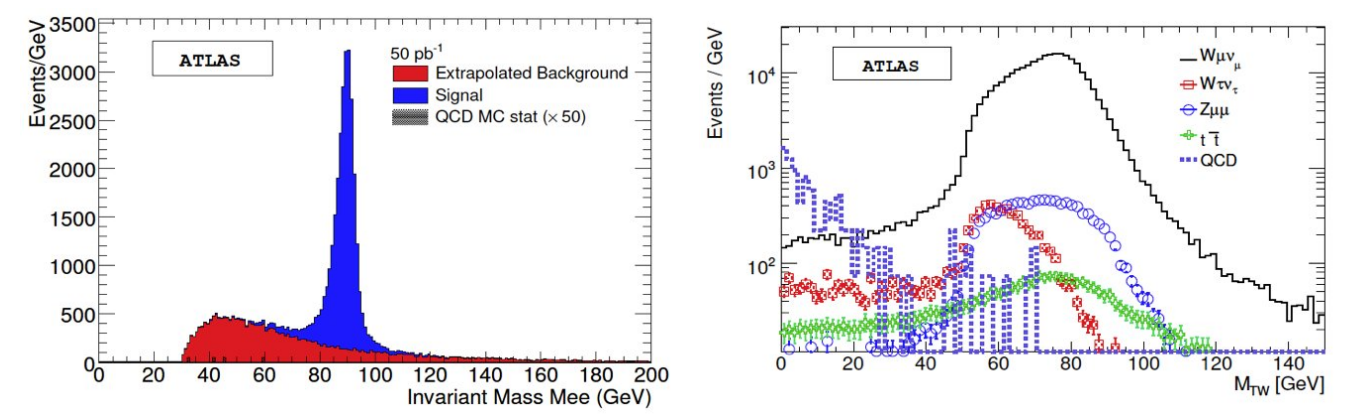

Figure 2: $\mathrm{W} / \mathrm{Z}$ mass spectra at the end of the described selection for $50 \mathrm{pb}^{-1}$ of simulated MC samples at $14 \mathrm{TeV}$. Left: invariant mass spectrum of two electrons coming from $\mathrm{Z}$, signal and background. Right: reconstructed $\mathrm{W}$ transverse mass spectrum, signal and backgrounds. 


\section{W lepton decay spectra to improve PDF knowledge}

It's possible to use $\mathrm{W}$ related measurements at LHC to improve our present knowledge of the proton structure function at low- $x$. In fig. 3 the decay lepton spectra and the lepton asymmetry are shown for the electrons coming from the $\mathrm{W}$ at generation level and after the detector simulation and selection, using three different PDF sets (CTEQ6.1, ZEUS-S and MRST2001) [4].

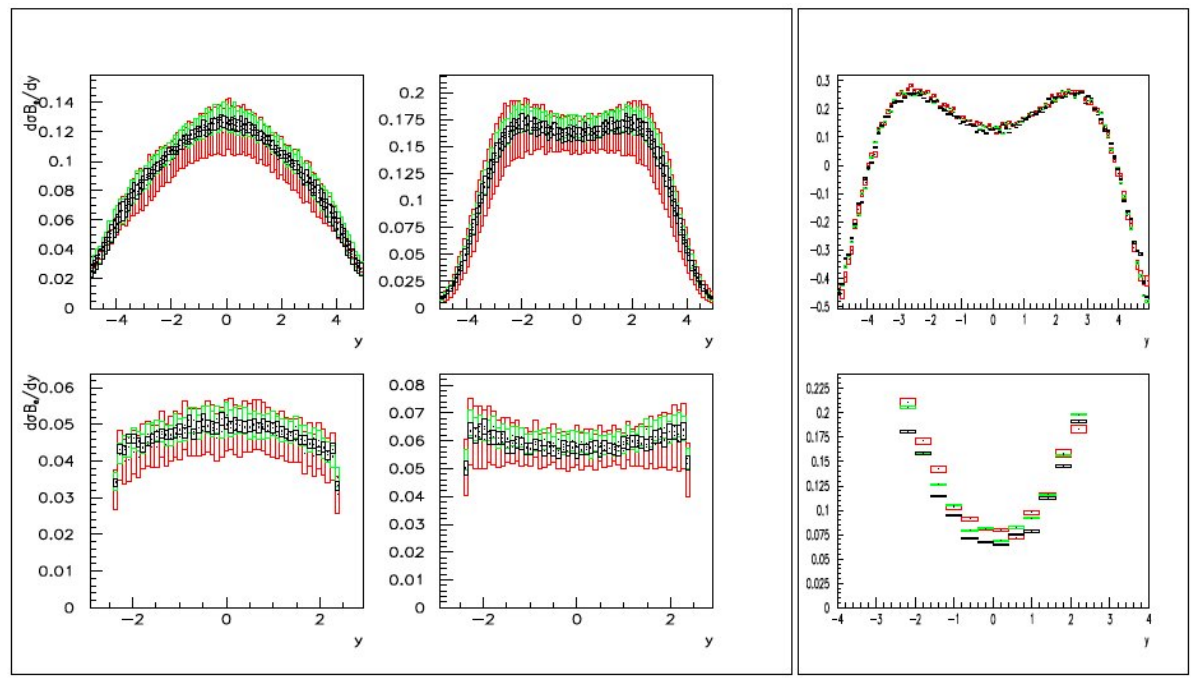

Figure 3: Top row: $\mathrm{e}^{-}, \mathrm{e}^{+}$and Asymmetry rapidity spectra for the lepton from the $\mathrm{W}$ decay, generated using CTE6.1 (red), ZEUS-S (green) and MRST2001 (black) PDF sets with full uncertainties. Bottom row: the same spectra after passing through the ATLFAST detector simulation and selection cuts.

A pseudo-experiment shows how is possible to use the decay lepton spectra to improve the current PDF description at low- $x$ if one is able to perform a measurement more accurate than $\sim 5 \%$ [4]. W pseudo-data events at $14 \mathrm{TeV}$ are simulated using a certain PDF set, CTEQ6.1 and the lepton spectra are reconstructed assuming $\sim 5 \%$ of experimental uncertainty. Then they are compared with the prediction of another PDF set, ZEUS-S (fig. 4 left panel). When the pseudodata are used to correct the new prediction the low- $x$ gluon shape parameter $\lambda\left(x g(x) \sim x^{-\lambda}\right)$ turns out to be shifted and the uncertainty is reduced, from $\lambda=-0.199 \pm 0.046$ to $\lambda=-0.181 \pm 0.030$ (fig. 4 right panel).

\section{Conclusion}

The Standard Model rediscovery has already started at LHC and the $\mathrm{W}$ and $\mathrm{Z}$ bosons are one of the first benchmark measurements. A broad range of W/Z related measurements will be feasible at $7 \mathrm{TeV}$ with $100 \mathrm{pb}^{-1}$ which means till the end of 2010. It has been shown how $Z \rightarrow l l$ can be used to measure from data lepton identification and trigger efficiencies as well as momentum scale and resolution and missing $E_{T}$ scale. A robust selection has been developed which allows to measure the $\mathrm{W} / \mathrm{Z}$ cross-section. Finally $\mathrm{W}$ and $\mathrm{Z}$ related measurements will be a unique opportunity to improve our knowledge of protonic structure in the unexploited low- $x$ regime, for example through measurement of the $\mathrm{W}$ lepton decay spectra. 

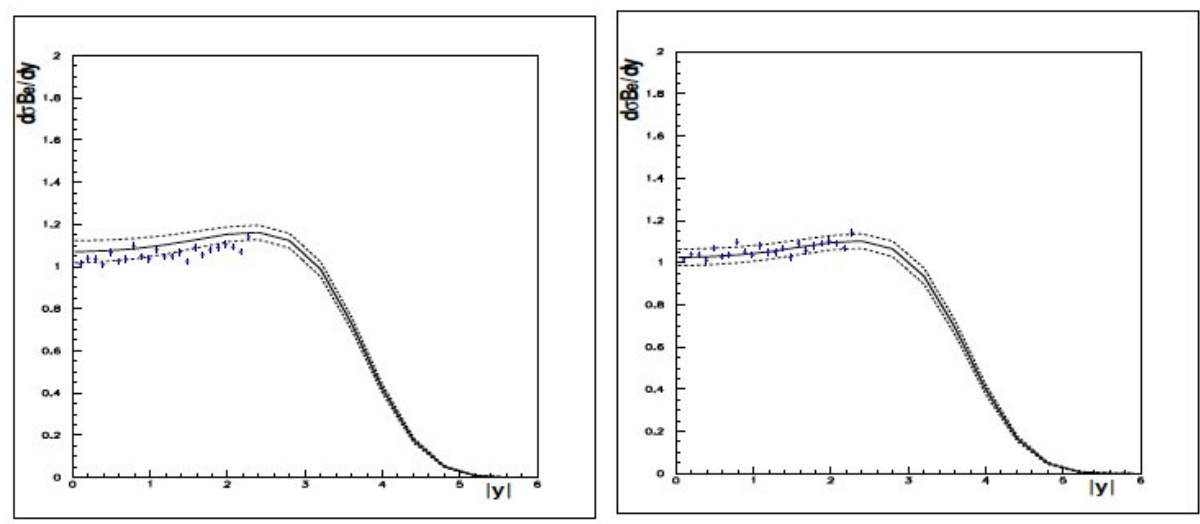

Figure 4: Left: $\mathrm{e}^{+}$rapidity spectra generated from CTEQ6.1 PDFs which have been passed through the ATLFAST detector simulation and corrected back to generator level using ZEUS-S PDFs (dots) compared to the analytic prediction using ZEUS-S PDFs (line). Right: the same lepton rapidity spectrum as above compared to the analytic prediction AFTER including these lepton pseudo-data in the ZEUS-S PDF fit.

\section{References}

[1] ATLAS Collaboration, CERN-OPEN-2008-020,arXiv:0901.0512

[2] A.Tricoli et al., ATL-PHYS-CONF-2005-008

[3] H1 and ZEUS Collaborations, Combined Measurement and QCD Analysis of the Inclusive ep Scattering Cross Sections at HERA, ariXiv:0911.0884

[4] A. Tricoli, A. M. Cooper-Sarkar and C. Gwenlan, HERA and the LHC - A workshop on the implications of HERA for LHC physics, CERN-2005-014, DESY-PROC-2005-001 arXiv:hep-ph/0601012v3 